\title{
Chondromyxoid Fibroma of Two Cervical Vertebrae with Involvement of Surrounding Soft Tissue: Radiologic Diagnostic Dilemma
}

\author{
Shokouh Taghipour Zahir ${ }^{1,{ }^{*}}$; Naser Sefidrokh Sharahjin ${ }^{2}$; Farzad Sadlu Parizi ${ }^{3}$; Koorosh \\ Rahmani $^{4}$ \\ ${ }^{1}$ Department of Pathology, Shahid Sadoughi University of Medical Sciences, Yazd, Iran \\ ${ }^{2}$ Department of Radiology, Shahid Sadoughi University of Medical Sciences, Yazd, Iran \\ ${ }_{4}^{3}$ Department of Neurosurgery, Shahid Sadoughi University of Medical Sciences, Yazd, Iran \\ ${ }^{4}$ School of Medicine, Shahid Sadoughi University of Medical Sciences, Yazd, Iran \\ *Corresponding author: Shokouh Taghipour Zahir, Department of Pathology, Shahid Sadoughi University of Medical Sciences, Yazd, Iran. Tel: +98-3518113615, Fax: +98-3518114100, \\ E-mail:shokouh_zahir@yahoo.com; taghipour@ssu.ac.ir
}

Received: April 10, 2014; Revised: July 7, 2014; Accepted: August 13, 2014

\begin{abstract}
Chondromyxoid fibroma is a rare benign cartilaginous neoplasm that mostly affects the metaphyseal region of the long bones. The tibia, small tubular bones of the foot, the distal femur and pelvis are common locations, but involvement of the vertebral bones, especially the cervical vertebra, is very rare. Radiographic features show typical characteristics and this tumor often presents as a lobulated, eccentric radiolucent lesion with no periosteal reaction. In addition, geographic bone destruction is seen in all cases. We present an adult female with a one-year history of neck pain, and ultrasound findings that suggest a right paravertebral muscular lesion due to inflammatory or neoplastic origins. The histopathological studies confirmed that the biopsied specimen was a chondromyxoid fibroma of the cervical vertebrae laminae and spinous processes (C3 and C4) with abutting soft tissue. Despite the unusual location and soft tissue presentation, a chondromyxoid fibroma should be considered in the differential diagnosis of a cervical bone lesion.
\end{abstract}

Keywords: Tissue; Chondromyxoid Fibroma; Vertebrae

\section{Introduction}

Chondromyxoid fibroma (CMF) is a rare benign cartilaginous neoplasm, and it accounts for less than $1 \%$ of all bone tumors. There is no gender predilection, but in some of the published case series, a male tendency has been reported (1-10). It occurs mainly in the second and third decades, and the majority occurs before the age of 30 years (2). The most common locations are the upper third of the tibia, small tubular bones of the foot, the distal femur and pelvis $(3,4)$. Involvement of the vertebral column is rare, especially in the cervical region. In general, CMFs originate from the metaphyseal region of the long bones. Literature reviews have shown that CMF is very rare in the cervical region, and only less than 11 cases have been reported up to now $(1,5-8)$. CMF of the cervical spine may present with unusual radiological findings and pose a diagnostic dilemma; therefore, we first report our case and then briefly discuss its radiological, histopathological and clinical characteristics.

\section{Case Presentation}

A 36-year-old woman came to the neurosurgery clinic with a one-year history of neck pain and semi-lateral radic- ular pain in her right hand. Her past medical history was unremarkable. On ultrasound examination, there was a hypo-echoic oval mass-like lesion located on the right side of the C3 and C4 vertebrae and adjacent subcutaneous soft tissue, measuring $1.2 \times 3.6 \times 1.6 \mathrm{~cm}$. The ultrasound possible differential diagnosis was right paravertebral muscle soft tissue lesion due to inflammatory or neoplastic origins. A lateral plain x-ray of the neck revealed an ill-defined hypoattenuated area within the deep soft tissue of the posterior aspect of the $\mathrm{C} 3$ and $\mathrm{C} 4$ vertebrae just posterior to the related spinous process without bony erosion, demineralization, foreign body, or soft-tissue calcification. Irregularity and possible erosion in the spinous process of $\mathrm{C} 5$ of the cervical vertebrae was seen (Figure 1).

On Doppler ultrasound, there was no blood flow detected and the mass was reported as a hypo-vascular lesion. On magnetic resonance imaging (MRI), a well-defined encapsulated $2.6 \times 2 \times 1.8 \mathrm{~cm}$ mass lesion emanating from the soft tissue of the paravertebral neck was detected that abutted the spinous process and lamina of the posterior neural arc of the C3 and C4 vertebrae and appeared to be low signal intensity on the T1 weighted imaging (T1W1)

Copyright (C) 2015, Tehran University of Medical Sciaaences and Iranian Society of Radiology. This is an open-access article distributed under the terms of the Creative Commons Attribution-NonCommercial 4.0 International License (http://creativecommons.org/licenses/by-nc/4.0/) which permits copy and redistribute the material just in noncommercial usages, provided the original work is properly cited. 
(Figure 2). The lesion was seen as a target shaped lesion with a centrally hypo-intense and peripherally mild hyperintense thick rim and surrounding edema on T2WI (Figure 3). On post-contrast T1WI, considerable enhancement of the peripheral thick rim and adjacent posterior soft tissues of C3 and C4 vertebrae were presented (Figure 4). The patient underwent surgical resection, and a frozen section study was requested. The specimen received for frozen section consisted of one piece of cream colored tissue measuring $3 \times 2 \mathrm{~cm}$. Histopathological examination revealed a lobulated tumoral lesion composed of fibromyxoid connective tissue admixed with small islands of cartilaginous tissue

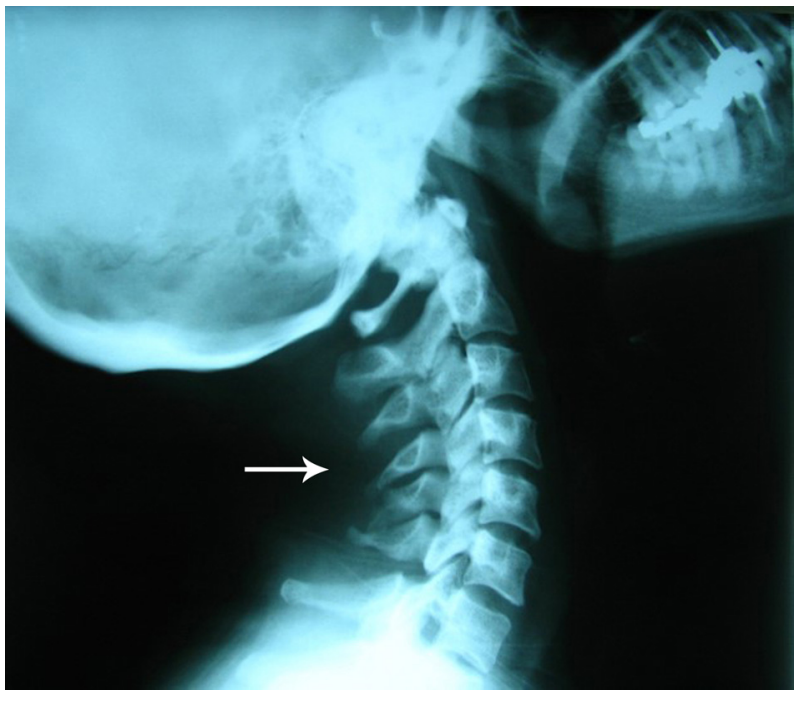

Figure 1. A 36-year-old woman with neck pain and semi-lateral radicular pain in the right hand. A lateral plain x-ray of the neck reveals an ill-defined hypoattenuated area adjacent to the deep soft tissue of the posterio aspect of the $\mathrm{C} 3$ and $\mathrm{C} 4$ vertebrae, just posterior to the related spinous process and some irregularity or possible erosion in the spinous process of fifth cervical vertebra.

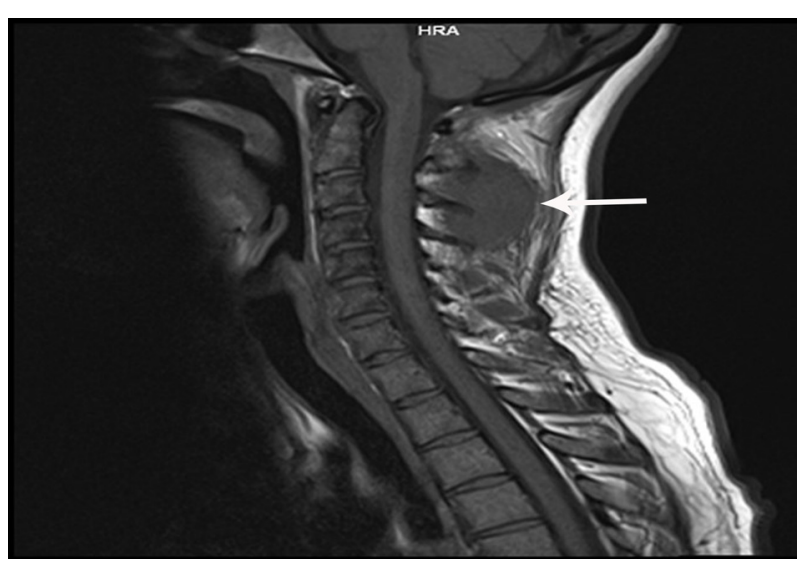

Figure 2. A well-defined encapsulated mass lesion emanating from the soft tissue of the paravertebral neck abutting spinous process and lamina of the posterior neural arc of the $\mathrm{C} 3$ and $\mathrm{C} 4$ vertebrae on sagittal $\mathrm{T} 1$ weighted MRI. The mass showed low signal intensity on sagittal T1WI. and areas of calcification, so the frozen section suggested a benign lesion. The received specimen for permanent diagnosis consisted of multiple pieces of brownish colored tissues altogether measuring $4 \times 3 \mathrm{~cm}$. Histopathological examination revealed a tumoral lesion composed of proliferated chondrocytes, admixed with fibromyxoid connective tissues, and scattered osteoclast-like multinucleated giant cells (Figure $5 \mathrm{~A}, 5 \mathrm{~B}$ ). Mature bone trabeculae rimmed by osteoblasts with areas of calcification were also detected. The final diagnosis was CMF of the posterior elements of the cervical bones with extension to the adjacent soft tissue. The patient received no other treatment and after two years of surgery, she has not suffered from any problem related to the lesion in her neck area and no recurrence was reported.

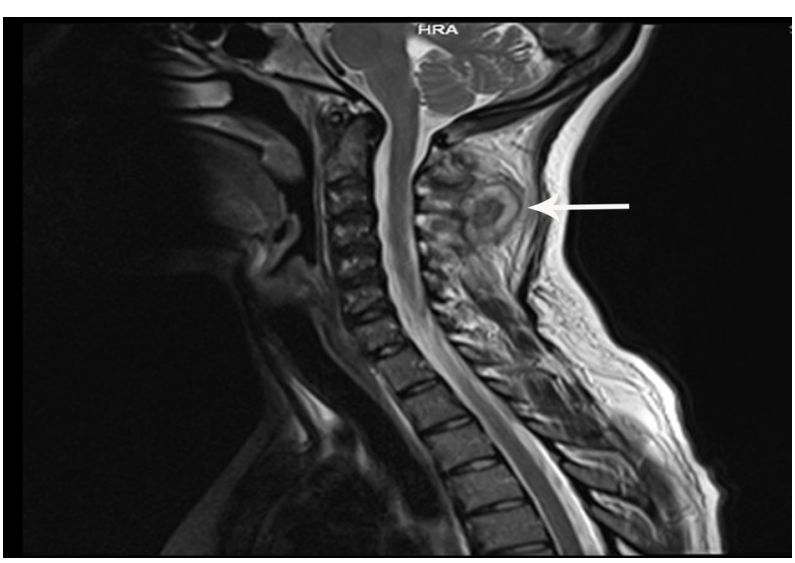

Figure 3. A target shaped lesion, central hypointense and peripheral mild hyperintence thick rim with surrounding edema located on the mid-line of the neck around C3 and C4 vertebrae on sagittal T2WI is seen.

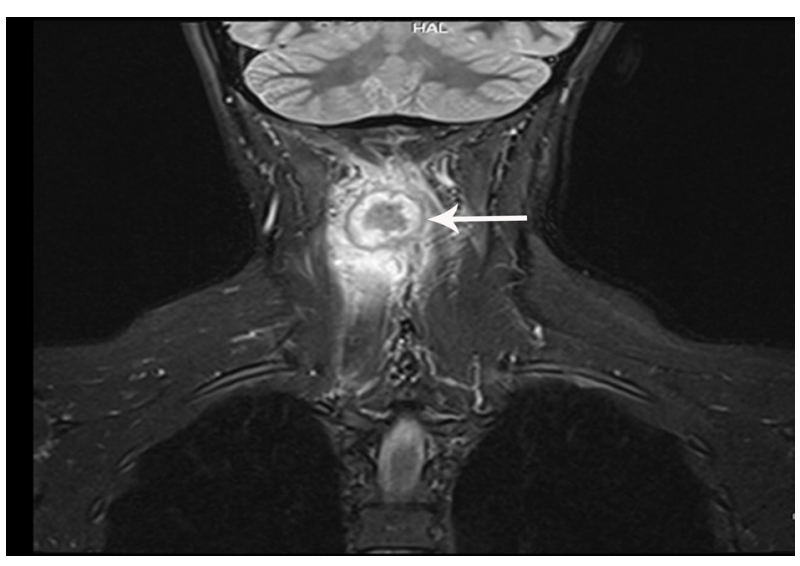

Figure 4. Coronal post contrast T1WI shows considerable enhancemen of the peripheral thick rim and adjacent posterior soft tissues of the $\mathrm{C} 3$ and $\mathrm{C} 4$ vertebrae. 


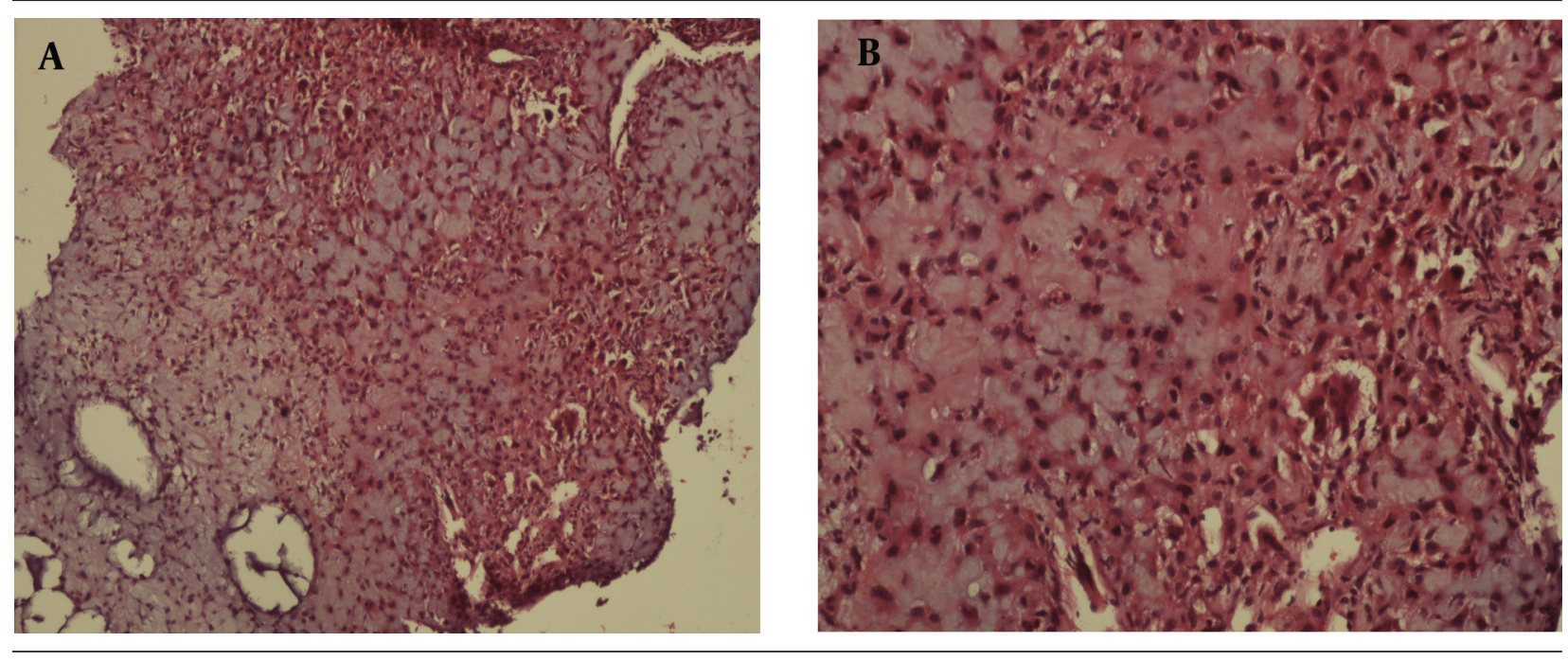

Figure 5. A and B, Histopathological examination shows chondroid tissue admixed with fibroblasts and scanty multinucleated giant cells (H \& E staining, Magnification, $\times 200$ and $\times 400$ ) spinous process of fifth cervical vertebra.

\section{Discussion}

CMFs are extremely rare benign cartilaginous bone neoplasms that were initially described by Jaffe and Lichtenstein in 1948 (10). There is no gender predilection, but in some of the published case series, higher numbers of males have been reported. It usually occurs in the second or third decades and the majority appears before the age of 30 years. Most of the CMFs are located in the metaphyseal region of the long bones. One third of them forms in the tibia, small tubular bones of foot, and the distal femur and pelvis (1-12). CMFs in the vertebral columns are rare, especially in the cervical region and related soft tissue vicinity. Lopez et al. reported CMF of the cervical spine in a 20-year-old man with neck pain that was located in the body of the C2 vertebra. X-ray showed a lytic lesion in the C2 body that causes instability of the craniocervical vertebra without extension into the soft tissue (5). Our case was an adult female who had suffered from a long history of neck pain and there was an imperceptible lump on an x-ray in the right paravertebral soft tissue. Although the radiological findings were not diagnostic for CMF, it originated from the lamina and spinous processes of the cervical bones and extended into the adjacent soft tissue. Spinal CMF was first reported by Benson and Bass (1). Radiological features are not characteristic, but may present as a lobulated, eccentric radiolucent lesion with no periosteal reaction, and in all of the patients, geographic bone destruction is visible. The margin of the CMF is sclerotic, and defined with pseudotrabeculation in $57 \%$ of the cases, and matrix calcification is seen in about $12.5 \%$ of the cases (11). MRI features of CMF of vertebral column are not specific, but the helpful features are the peripheral intermediate signal band, central high signal intensity on T2-WI, and peripheral nodular enhancement or central non-enhanc- ing focus on contrast-enhanced T1-WI (12). In addition, a doughnut sign is seen in bone scans of CMFs; however, this is a non-specific finding because it may be found in other bone tumors $(11,12)$. Other benign tumors, such as aneurysmal bone cyst, giant cell tumor, enchondroma, non-ossifying fibroma and chondroblastoma could be listed in the differential diagnoses. In long bones, CMF originates from the metaphysis close to the physis, and it may also extend into the epiphysis or diaphysis. Furthermore, it usually appears as an eccentric, oval shape lesion. In small bones, a CMF may involve the diaphysis, metaphysis, and epiphysis (1). The preferred imaging study is conventional radiography with its specific characteristics composed of a bubbly cystic appearance, but a computed tomography (CT) scan displays a sclerotic calcification margin and trabeculation better than conventional radiography (11). In our case, a plain X-ray showed a barely discernible ill-defined soft-tissue density within the deep soft tissues of the posterior aspect of the $\mathrm{C} 3$ and $\mathrm{C} 4$ vertebrae just posterior to the related spinous process without characteristic features of CMF in the vertebral bones. CMFs have different tissue components with a heterogeneous appearance on MRI studies. For example, chondroid and myxoid tissues create an intermediate to high signal on proton-density and T2-WI or low signal on T1-WI (12). CMFs show a high accumulation of fluorodeoxyglucose in positron emission tomography (PET) scan, but we did not perform this study for our patient. The most common site of vertebral column involvement by CMF is in the thoracic region, with a predilection for the posterior neural arc (4). Chondrosarcoma, osteoblastoma, aneurysmal bone cyst, giant cell tumor of the bone, metastasis, multiple myeloma, and collapsed hemangioma of the vertebral bodies are 
the most common differential diagnoses in radiological investigations. Jonathan et al. reported CMF in the seventh cervical vertebra of a 35-year-old man, in whom Xray, MRI and bone scans, suggested an aneurysmal bone cyst or a giant cell tumor (1). Two adjacent vertebral bone involvements, especially in the cervical vertebrae have not been reported yet. Bruder et al. reported a case of CMF in two thoracic vertebrae that originated from vertebral bodies, but our case was CMF in two cervical vertebrae with involvement of the laminas and spinous processes (13). The symptoms of this benign tumor seem to be progressive longstanding pain, swelling, and motion restriction of the affected limb (1-8). Histopathologically, the tumor is composed of chondroid, myxoid, and fibrous connective tissue, which are arranged in a lobular pattern. Scattered osteoclast-like multinucleated giant cells are occasionally seen inside the tumor, especially at the periphery. Areas of calcification are also present in some cases. Mitotic figures are rare or absent (10). The main differential diagnosis of CMF from chondrosarcoma histologically is the sharp borders of the lobules and also radiologic studies which could help to make the correct diagnosis (11). En bloc excision of the tumor is the treatment of choice, but recurrences after curettage have been reported. Our case has not had any recurrence after the excision.

Although CMF is a rare benign bone tumor, involvement of the vertebral column especially the cervical vertebrae is very rare, but we listed it in the differential diagnosis despite the unusual soft tissue extension. Conventional radiology with MRI and PET scan could give us better diagnostic information.

\section{Acknowledgements}

The authors would like to thank Dr Aghili for radiology report guidance.

\section{Authors' Contributions}

All authors have participated equally in the present study.

\section{Funding/Support}

The study was self-funded.

\section{References}

1. Jonathan A, Rajshekhar V, Chacko G. Chondromyxoid fibroma of the seventh cervical vertebra. Neurol India. 2008;56(1):84-7.

2. Bala A, Robbins P, Knuckey N, Wong G, Lee G. Spinal chondromyxoid fibroma of C2.J Clin Neurosci. 2006;13(1):140-6.

3. Gutierrez-Gonzalez R, De Reina L, Saab A, Jimenez-Heffernan J, Garcia-Uria J. Chondromyxoid fibroma of the lumbar spine: case report and literature review. Eur Spine J. 2012;21 Suppl 4:S458-62.

4. Kikuchi F, Dorfman HD, Kane PB. Recurrent chondromyxoid fibroma of the thoracic spine 30 years after primary excision: case report and review of the literature. Int J Surg Pathol. 2001;9(4):323-9.

5. Lopez-Ben R, Siegal GP, Hadley MN. Chondromyxoid fibroma of the cervical spine: case report. Neurosurgery. 2002;50(2):409-11.

6. Crocker M, Corns R, Bodi I, Zrinzo A, Gleeson M, Thomas N. Chondromyxoid fibroma of the skull base invading the occipitocervical junction: report of a unique case and discussion. Skull Base. 2010;20(2):101-4.

7. Sfreddo E, Falavigna A, Soares VB, Nesi A, Nascimento V, Silva P Chondromyxoid fibroma of C1: first case report. Coluna/Columna. 2012;11(1):84-6.

8. Standefer M, Hardy Jr RW, Marks K, Cosgrove DM. Chondromyxoid fibroma of the cervical spine-a case report with a review of the literature and a description of an operative approach to the lower anterior cervical spine. Neurosurg. 1982;11(2):288-92.

9. Subach BR, Copay AG, Martin MM, Schuler TC, Romero-Gutierrez M. An unusual occurrence of chondromyxoid fibroma with secondary aneurysmal bone cyst in the cervical spine. Spine J. 2010;10(2):e5-9.

10. Jaffe HF. Chondromyxoid fibroma of bone.: A distinctive benign tumor likely to be mistaken for chondrosarcoma. Arch Pathol. 1948;45:541-51.

11. Wilson AJ, Kyriakos M, Ackerman LV. Chondromyxoid fibroma: radiographic appearance in 38 cases and in a review of the literature. Radiology. 1991;179(2):513-8.

12. Kim HS, Jee WH, Ryu KN, Cho KH, Suh JS, Cho JH, et al. MRI of chondromyxoid fibroma. Acta Radiol. 2011;52(8):875-80.

13. Bruder E, Zanetti M, Boos N, von Hochstetter AR. Chondromyxoid fibroma of two thoracic vertebrae. Skeletal Radiol. 1999;28(5):286-9. 